\title{
Non-Formal education in astronomy: The experience of the University the Carabobo
}

\author{
Nelson Falcón \\ Dpto. de Fisica. FACYT, University the Carabobo, \\ Apto. Postal 129 Avda Bolivar Norte, Valencia 2001, Carabobo, Venezuela \\ email: nelsonfalconv@gmail.com
}

\begin{abstract}
Since 1995, the University the Carabobo, in Venezuela, has come developing a program of astronomical popularization and learning Astronomy using the Non formal education methods. A synopsis of the activities is presented. We will also discuss some conceptual aspects about the extension of the knowledge like supplementary function of the investigation and the university teaching. We illustrate the characteristics of the communication with an example of lectures and printed material. The efficiency of the heuristic arguments could be evaluated through a ethnology study. In that order of ideas, we show some images of the activities of astronomical popularization. We can see the population and great concurrence with chronological (and cultural) heterogeneity. We conclude that the Non formal education, structured with characteristic different to the usual educational instruction, constitutes a successful strategy in the diffusion and the communicating astronomy.
\end{abstract}

Keywords. Communicating astronomy, Non formal education, Learning Astronomy Methods.

\section{Introduction}

For specialists is obvious the presence and influence of astronomy in daily life in the cultural development of mankind, but that vision is not sufficiently understood. The study of astronomy for the general people is very important (Stavinschi 2006), because we questions about the nature and concept of humanity, to inquire about their place and role in the vast universe that surrounds our planet and our awareness of the finitude of life, in the cycle of continous movements of the stars. In all cultures one of the first activities related to the stars, has been the determination of the time (so far as its registration) and the making of calendars (with which, for example, setting the dates so propitious for crops as for the holidays). Any international effort to promote astronomy world wide today must necessarily take into account its cultural and historical component. The past few decades have ushered in an age, which we may call the Age of Cultural Copernicanism. Cultural Copernicanism would imply that no cultural or geographical area, or ethnic or social group, can be deemed to constitute a superior entity or a benchmark for judging or evaluating others (Kochhar 2006). Also, other of the most fascinating aspects of astronomy is its interaction with other sciences, particularly physics and mathematics, space is an excellent place to verify the universality of physical laws in our planet and its generalization test one of the objectives of Astronomy. Thus the astronomy as auxiliar science and humanizing knowledge humanizing may be cause that teaching Astronomy is more demanded is non-formal education (Kubinec 1995). Non-formal education (NFE) is defined as any educational activity, organized and systematically conducted outside the official sphere, to facilitate certain types of learning to particular subgroups of the population (Trilla 1999). Its organization is based on independent units. The accreditation is specific to special accomplishments, not for levels modalities, career or academic degrees. 
Here education providers often are neither academics nor professionals. May be, in the case of astronomy, amateur astronomers and physicists, who together with professional astronomers and researchers, provide general knowledge of astronomy to large segments of the population. On this basis, four forms of NFE can be distinguished by reference to their relationship with the formal school and college system. These are: paranormal education, popular education, education for personal development and professional training. They constitute four relatively autonomous segments within the educational field which differ from each other because they: respond to different educational needs; serve different clienteles; are being organized by different educational agencies; and, above all, have different relationships with the formal educational system. Knowledge and skill acquisition by direct imitation is now recognized among the opportunities of individual, group, organized and spontaneous learning and is no longer reserved to the area of primary socialization. There are attempts to make it more institutionalized e.g. in the eases of amateur artistic creative activity or of the situational approach to language learning. This process goes together with the rediscovery of certain modes of learning which had not previously been recognised as part of the dominant European cultures (e.g. intuitive learning, role acquisition based on empathy, etc.).In the case of the most rapidly growing technologies, there is practically no better way than learning by direct inter-personal imitation (and learning by trial and error). The dramatic rise in the popularity of learning how to use personal computers or basic astronomy concepts are conspicuous example of this. Also the NFE in the social-cultural life sphere, quite frequently the process of learning itself is more important than the result; learning as an activity becomes more important than the knowledge acquired (La Belle 1982). Taking part in some collective learning-teaching experience is one way of searching for a sense of community. In the case of education of the elderly, emphasis is laid on the mental hygiene effect of the learning activity itself. In recent years, non-formal education has been applied successfully in some countries as a method of popularization of astronomy in basic school (Pomares 2001), stars workshop (Hernán-Obispo 2005) and, also, in the search for extraterrestrial intelligence (Bhathal 2001).

\section{Overview: Non formal education in astronomy}

The popularization (Non formal education) of the Sciences, in Astronomy specifically, is conceived like a differentiated activity, permanent, generic and with effect multiplier. - Differentiated. The popularization is distinguished of the public culture (all public), understanding this as a complementary formation for the people sector with level of knowledge means in science (students of secondary education or professionals without specific formation in Physical sciences, for example lawyers, administrators, etc.).

- Permanent. It is conceived that the dynamic process of the Sciences requires a permanent bring up to date and integrative vision, even for those who have reached levels of specific knowledge in areas of the sciences and astrophysics.

- Generic. We understands that the popularization is different to the Instruction, in the sense that the popularization attempts the information in the daily language, while the Instruction emphasizes the systematizing, by means of the technical and symbolic language.

- Effect multiplier. The non formal education, opposite at the instruction, is essentially motivational and in such a sense it impels the participants to act as spokesmen, it is worth to say that the knowledge was transformed into news and ideas.

The non formal Education with the amateurs and public should be structured with different characteristics to the usual educational instruction, as Festive atmosphere., Leisure 
and bohemian activities, Public places, Party: Share together the play, Discusion general: direct and informal speech to speak, Universal character of astronomy, Relationship with the environment and local cultural and ethnology and finally Integration of Science, Technology and astronomy literature, poetry, music and folklore.

Also, we think that the non formal Education should be structured with characteristic different to the usual educational instruction, such as - Avoid numeric data and Symbolic language. - Emphasize the use of similes and inferences. - The phenomenon was first and then the theoretical principles. - Define concepts and technical terms. - Using anecdotes and to create expectations to the reader. - Emphasis on cognitive processes and not on the historical evolution of concepts - Indicating why and where to obtain more information.

\section{The experience of the University the Carabobo}

The importance of the popularization of the Science in general, and more particularly of the Astronomy, it has constituted one of the objectives in the novel Physics Department of the Experimental Faculty of Sciences and Technology (FACYT) of the Carabobo University. Since their creation, June 04 of 1995, the FACYT has intended as goal the reception of students of sciences and the relationship with the social environment of the city (Valencia, Edo Carabobo Venezuela). In this order of ideas, the Department of Physics has come developing a program of astronomical popularization (Non formal education). The Carabobo University is conformed by 60.000 regular students and 3500 Professor and Researcher,, contained in seven Faculties: Health Sciences, Education Sciences, Engineering, Economy and Social Sciences, of Laws, Dentistry, and Science and Technology. One of the objective of the FACYT is to develop extension activities toward the community, constituting a center of consultantship, of information and popularization of results of the scientific and technological investigation developed in the country. Their influence environment embraces the city of Valencia (with approximately a million inhabitants) and an outlying population of another million of dispersed inhabitants in five federal entities (States). The effectiveness of the popularization of the astronomy depends essentially on the effectiveness of the communication, as the reach of the communication in the local population, depends on the empathy of the message with the fellow. Non formal education constitutes a successful strategy in the diffusion of the message (information). The speech should be structured with characteristic different to the usual educational instruction. Also the same technique would be employed in diverse media, for example: programs of radio and TV (in the University radio, FM104.5 coverage state and 24 hours of continuous transmission, as well as its own local television channel, UC-TV, Channel 54 UHF transmission), chats (in classrooms, conferences, seminars and forums) or in written means (billboards, press articles, etc.). Since 1995, the FACYT employed those heuristic arguments, non formal education, for the astronomy teaching. It have been generated more of fifty articles for the written press, dozens of radial programs and TV programs.

We illustrate the characteristics of the communication and NFE strategy with an example of lectures and printed material, the efficiency of the heuristic arguments could be evaluated through a ethnology study (Falcon et al. 2005). An example of written communication in non formal education. (i) Suggestive Tittle and Picture, (ii) Introduction: provocative, disturbing sentence or questions, (iii) Narrative: phenomenons or history, (iv) Description of the general idea, followed by the details and data, (v) Close or final: 


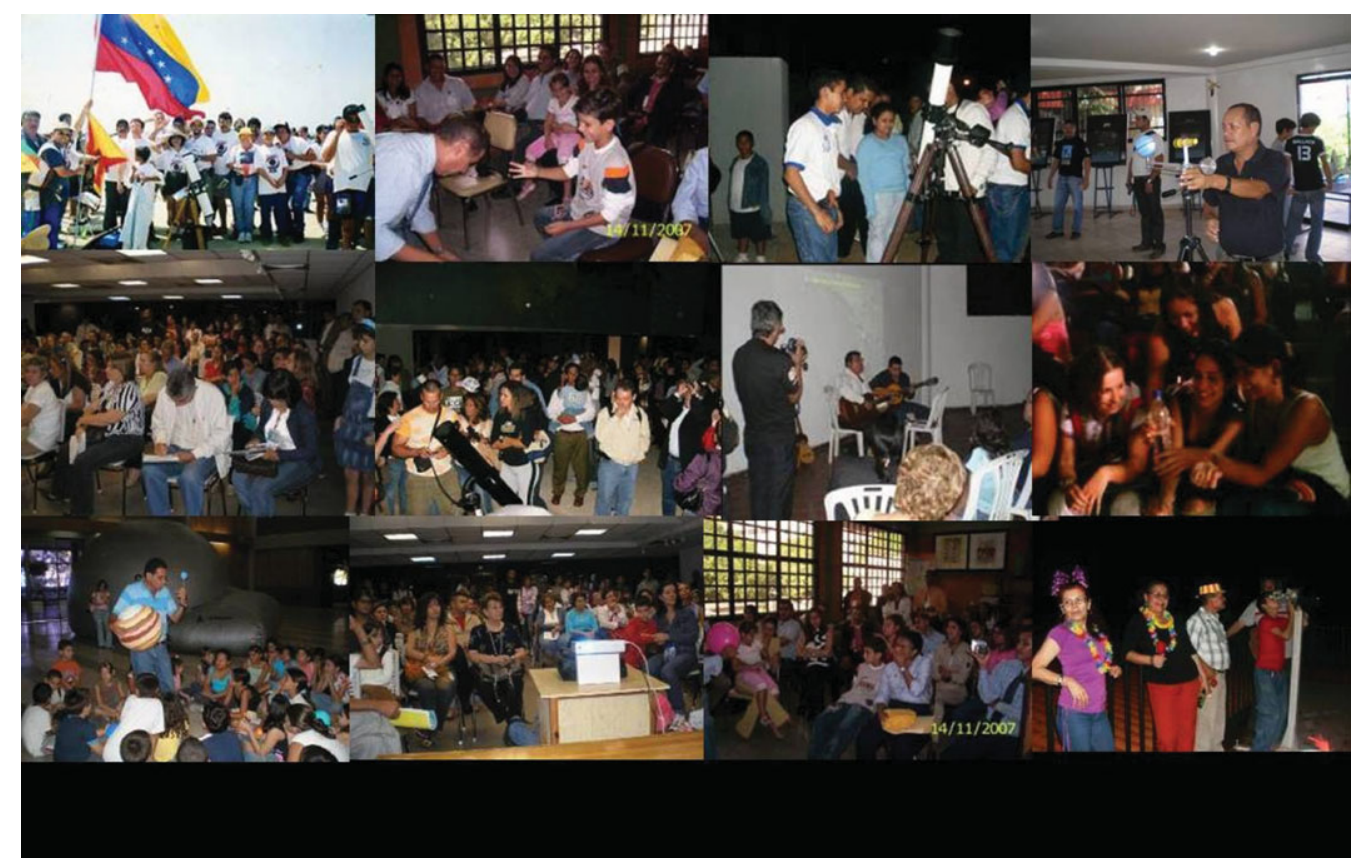

Figure 1. We can see the population and great concurrence and the chronological (and cultural) heterogeneity.

linking of the topic with local reality, astronomical anniversary and some thing of present time.

All activities take place in communities, in public places such as parks, schools, theaters, highschools, amphitheatres and boulevards. They generally combine lectures, seminars, demonstrations of experimental physics and telescopic observation with the music, stories, festivals, dances, drama and poetry readings. Integrating science as another aspect of culture.

In that order of ideas, we show some images of the activities of astronomical popularization in next figure.

Can we reach in better ways impaired or confined communities? Since 1995, the FACYT employed those heuristic arguments, non formal education, for the astronomy teaching and the reception of students of sciences and the relationship with the social community. The effectiveness of the popularization of the astronomy depends essentially on the effectiveness of the communication. We show some images of the activities of astronomical popularization. We can see the population's great concurrence and the public's heterogeneity (chronologica and cultural). There are general public not University stundent. Thus we think that the non formal Education maybe a better way by the public astronomy instruction.

Can collaborations be improved with amateurs and be more cooperative with their activities, along with those offered by the planetaria world-wide? To make this extensive program of activities (activities NFE), the University of Carabobo an agreement of cooperation with local societies and clubs of amateur astronomy (since 1996 ). This agreement between the amateur and professional astronomers is a legal basis that allows the use of common facilities and teaching resources, and joint public activities in NFE, also notice that all activity are free of charge and voluntary. More information in web site www.sociedadastronomica.uc.edu.ve, asoaca.webcindario.com. 


\section{Implications}

What are the current efforts in astronomy education across the planet, especially in developing countries? One of the objectives of the Carabobo University is to develop extension activities toward the community, constituting a center of consultantship, of information and popularization of the science. Thus the Department of Physics has come developing a program of astronomical popularization (Non formal education) heuristic based on the principles that The reach of the communication in the local population, depends on the empathy of the message with the fellow. Non formal education constitutes a successful strategy in the diffusion of the message (information). The same technique would be employed in diverse media, for example: programs of radio, chats (in classrooms, conferences, seminars and forums) or in written means (billboards, press articles, etc.).

Also, particular approach to not formal Education in Astronomy, includes not only indigenous and folkloric astronomical knowledge, but also the culture of transmission of such knowledge One way of developing effective communication in astronomy is to combine the knowledge of the scientist with the most spectacular TV production integrating, through commentary and analysis, astronomy and science into the public debate of lay citizens significant cultural agenda. Connected with the ideas, orbital performance, of the synthetic arts, combining a knowledge of the truly spiritual as well as the universal, as Erwin Schrodinger said:

"Scientific knowledge forms part of the idealistic background of human life which exalted man from a nude and savage state to true humanity."

\section{References}

Bhathal, R. 2001, in The Search for Extrterrestrial Intelligence (SETI) in the Optical Spectrum III, S. A. Kingsley \& R. Bhathal (eds), Proc. SPIE, 4273, 240

Falcón, N. et al. 2005, in Communicating astronomy, T. J. Mahoney (ed) (La Laguna: IAC), p. 159

Hernán-Obispo, M. et al. 2005, EAS Publications Series, 16, 209

Kochhar, R. 2006, in Astronomy for the Developing World, 26th meeting of the IAU, Special Session 5, p. 4.

Kubinec, W. 1995. Bulletin of the American Astronomical Society, 27, 887

La Belle, T. J. 1982. International Review of Education, 28, 159

Pomares, O. 2001, in Astronomy for developing countries. Proceedings of a Special Session of the XXIV General Assembly of the International Astronomical Union, A. H. Batten (ed) (San Francisco: Astronomical Society of the Pacific), p. 163

Stavinschi, M. 2006, Innovation in Teaching/Learning Astronomy Methods, 26th meeting of the IAU, Special Session 2, 11

Trilla, J. 1999, Revista galega do ensino, 24, 199 